\title{
Motivação de pessoas idosas para a prática de atividade física: estudo comparativo entre dois programas universitários - Brasil e Portugal
}

\author{
Motivation of elderly people to engage in physical exercising: a comparative study between two \\ university-based programs - Brazil and Portugal
}

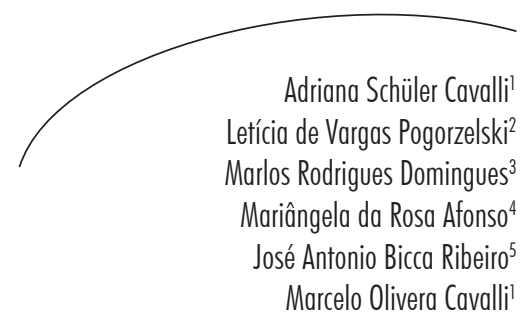

Resumo

Objetivo: Identificar o que motiva o idoso a participar em programas de atividade física em duas universidades conveniadas, uma no Brasil e outra em Portugal. Métodos: O estudo caracterizou-se como descritivo transversal de caráter quantitativo. A amostra foi composta por 263 participantes de ambos os sexos, com média de idade de 69,8 $\pm 6,44$ anos. Destes, 213 participavam do Núcleo de Atividades para a Terceira Idade da Escola Superior de Educação Física da Universidade Federal de Pelotas-RS, Brasil, e 50 participavam do Projeto Exercício e Saúde: Envelhecimento Ativo, da Faculdade de Desporto da Universidade do Porto, Portugal. Para a coleta dos dados, foi aplicado um questionário sobre características sociodemográficas e determinantes da prática de atividade física. $\mathrm{Na}$ análise estatística, foi utilizado o teste $t$ Student para amostras independentes e o valor de confiança de $\mathrm{p}<0,05$ para calcular a diferença das médias entre os grupos. Resultados: Quanto aos motivos de adesão aos projetos, houve diferença significativa para "indicação médica" ( $p<0,001)$, “recuperação de lesões" $(p=0,003)$, "ocupação do tempo livre" ( $p<0,001)$ e "motivos estéticos" ( $p=0,001)$. Quanto à importância da atividade física, houve diferença para "recuperação de lesões" ( $p=0,002)$ e "motivos estéticos" $(p<0,001)$. Diferenças significativas relacionadas tanto a variáveis sociodemográficas quanto aos determinantes para a prática de atividade física entre os grupos foram identificadas. Conclusões: Mesmo vivendo em diferentes realidades culturais, os idosos analisados compartilham os mesmos objetivos e motivação para a prática de atividade física, percebendo a saúde como uma preocupação em comum.

\footnotetext{
Escola Superior de Educação Física, Grupo de Pesquisa e Estudos Sociológicos em Educação Física e Esporte. Universidade Federal de Pelotas. Pelotas, RS, Brasil.

2 Escola Superior de Educação Física. Universidade Federal de Pelotas. Pelotas, RS, Brasil.

3 Escola Superior de Educação Física, Programa de Pós-Graduação em Educação Física. Universidade Federal de Pelotas. Pelotas, RS, Brasil.

4 Escola Superior de Educação Física, Programa de Pós-Graduação em Educação Física, Grupo de Pesquisa em Educação Física e Educação. Universidade Federal de Pelotas. Pelotas, RS, Brasil.

5 Faculdade de Educação. Programa de Pós-Graduação em Educação, Grupo de Pesquisa e Estudos Sociológicos em Educação Física e Esporte, Grupo de Pesquisa em Educação Física e Educação. Universidade Federal de Pelotas. Pelotas, RS, Brasil.
}

\section{Palavras-chave:}

Envelhecimento. Atividade Motora. Motivação. Relações Comunidade-Instituição. Idoso. 


\section{Abstract}

Objective: The study aimed to identify what makes elderly people engage in physical exercising (PE) programs in two fellow universities, in Brazil and Portugal. Methods: The study has a descriptive design and the sample is composed of 263 subjects of both genders with a mean age of $69.8 \pm 6.44$ years old. Of those, 213 participate in the "Núcleo de Atividades para a Terceira Idade" (NATI), School of Physical Education, Federal University of Pelotas, state of Rio Grande do Sul, Brazil; and 50 subjects take part in the "Projeto Exercício e Saúde: Envelhecimento Ativo" (PESEA), Faculty of Sport, University of Porto, Portugal. Data was gathered through a questionnaire specifying socio-demographic characteristics and determinants for PE. For the statistical analysis we used the $t$ Test for independent samples and the confidence value of $\mathrm{p}<0.05$ to calculate the mean difference between groups. Results: Regarding the reasons for adhesion, significant differences were found for "medical prescription" $(\mathrm{p}<0.001)$, "injury recovery" ( $\mathrm{p}=0.003)$, "way of pastime" $(\mathrm{p}<0.001)$, and "aesthetic reasons" $(\mathrm{p}=0.001)$. As for the importance regarded to PE, a difference for "injury recovery" $(p=0.002)$ and "aesthetic reasons" $(p<0.001)$ was detected. Significant differences related to socio-demographic variables and to determinants for PE were found among the groups. Conclusions: Even living in different social contexts, the elderly demonstrated to share the same objectives and reasons for engaging in PE programs, perceiving health as a common concern.
Key words: Aging. Motor Activity. Motivation. Community-Institutional Relations. Elderly.

\section{INTRODUÇÃO}

O envelhecimento da população é um assunto de interesse mundial, que demanda criação e manutenção de alternativas que viabilizem a permanência do idoso na sociedade, por meio da otimização de suas capacidades e da disponibilidade, principalmente, de serviços de saúde, segurança, previdência social e educação de forma continuada. ${ }^{1}$

No processo de envelhecimento, observamse diminuição das funções imunológicas do indivíduo, além de declínio nas capacidades funcionais e, consequentemente, alteração nos aspectos psicológicos envolvidos e decorrentes destas perdas graduais e contínuas. ${ }^{2}$ Pode ainda ser verificada diminuição significativa da atividade motora, resultante, em parte, da degeneração fisiológica, fatores ambientais, exigências da tarefa, doenças, estilo de vida ou a combinação desses elementos. ${ }^{3}$

$\mathrm{Na}$ tentativa de minimizar o impacto do processo de envelhecimento na vida diária dos idosos, a Organização Mundial da Saúde (OMS) argumenta que "[...] os países podem custear o envelhecimento se os governos, as organizações internacionais e a sociedade civil decretarem políticas e programas de envelhecimento ativo". A literatura sustenta que um estilo de vida ativo pode melhorar as funções mentais, sociais e físicas das pessoas que envelhecem. $\cdot^{4-6}$ Entretanto, a intenção de realizar um exercício físico depende, de acordo com Shephard, ${ }^{5}$ da avaliação ponderada do indivíduo entre a atividade/ação a ser executada e seu produto final resultante. Sendo assim, pesquisar os motivos que levam o indivíduo a realizar exercícios físicos pode encaminhar de forma efetiva a entrada e permanência destes em programas baseados no movimento.

Para um indivíduo ingressar num programa de atividade física, este encontra algumas barreiras principalmente relacionadas à motivação interna. Ao buscar a motivação necessária para tal mudança no estilo de vida, passa por estágios de acordo com o modelo transteorético que vão desde o desconhecimento dos benefícios da prática ("estágio de pré-contemplação"), 
onde estão as maiores barreiras, até o estágio de "manutenção", onde a atividade física foi incorporada em seu cotidiano, tornando-se um hábito. Entre esses estágios, existem outros que permeiam este processo, como o estágio de "contemplação", em que os efeitos e benefícios da atividade física são conhecidos, mas não há a prática efetiva. Existe, ainda, o estágio de "preparação", quando se percebem mudanças no comportamento do indivíduo, pois este começa a praticar as atividades, porém sem regularidade. Além disso, ainda existe o estágio de "ação", no qual o indivíduo tem a motivação necessária para mudar seu estilo de vida, tornando-se mais ativo.?

No caso dos idosos, a adoção de um estilo ativo nem sempre é observada, pois a grande maioria não tem a motivação necessária para ingressar num programa de atividade física, seja por fatores externos de ordem estrutural e física, ou por fatores internos de ordem psicológica. ${ }^{8}$ Tanto no Brasil como em outros países, estudos com a população idosa mostram grande prevalência de pessoas que são consideradas sedentárias ou inativas. ${ }^{9-11}$

Nesse contexto, destacam-se o papel e a importância das instituições de ensino superior, públicas e privadas, no que se refere à produção de conhecimento científico e propagação do mesmo com responsabilidade social, promovendo ações e realizando intervenções junto à comunidade, por meio de programas sociais. ${ }^{12}$ Este estudo teve como objetivo identificar o que motiva os idosos a participarem de programas de atividade física desenvolvidos por duas universidades conveniadas em diferentes contextos culturais, uma no Brasil e outra em Portugal.

\section{METODOLOGIA}

O estudo foi descritivo transversal, de caráter quantitativo. A amostra foi composta por 263 participantes (60 a 89 anos), de ambos os sexos, sendo 213 do Núcleo de Atividades para Terceira Idade (NATI), da Escola Superior de Educação Física da Universidade Federal de Pelotas (Brasil), e 50 do Projeto Exercício e Saúde: Envelhecimento Ativo (PESEA), da Faculdade de Desporto da Universidade do Porto (Portugal).

A seleção da amostra se deu de forma intencional, sendo que todos os idosos foram convidados a participar da pesquisa. Como critérios de inclusão, os indivíduos deveriam ter 60 anos de idade ou mais, estar devidamente cadastrados nos respectivos projetos e estar presentes na data da coleta dos dados, realizada no segundo semestre de 2010. Todos os idosos que aceitaram fazer parte da amostra realizavam aulas de hidroginástica, ginástica e/ ou musculação.

Para a coleta de dados, foi utilizado um instrumento, desenvolvido pelo Grupo de Pesquisa e Estudos Sociológicos em Educação e Esportes (GPES), da Universidade Federal de Pelotas, para delinear o perfil dos idosos participantes dos projetos. No respectivo instrumento, formado por questões fechadas, constavam informações como sexo, idade, país, tipo de atividade física praticada, além de uma questão na qual os idosos deveriam assinalar os motivos de participação nas atividades. $\mathrm{O}$ instrumento foi desenvolvido com o objetivo de caracterizar os idosos que usufruem das atividades do projeto. Além disso, os motivos de participação elencados e a importância atribuída à prática de atividade física servem de embasamento para o planejamento das atividades a serem desenvolvidas.

Para esta etapa, foi solicitada autorização de cada instituição responsável pelos projetos e seus respectivos coordenadores. Mediante autorização, cada idoso que aceitou participar do estudo recebeu um questionário para preenchimento, sendo que a aplicação ocorreu num dia de aula convencional, no horário das respectivas atividades praticadas nos projetos. Durante as coletas, os monitores do projeto auxiliaram no preenchimento dos instrumentos, esclarecendo as dúvidas que viessem a surgir.

Os dados foram digitados no software MS Excel e as análises estatísticas foram feitas no 
software Stata/SE 10.0. Para calcular a diferença das médias entre os grupos, utilizou-se o teste $t$ Student para amostras independentes e o valor de confiança aceito foi $\mathrm{p}<0,05$.

O estudo está de acordo com a Declaração de Helsinque e foi aprovado pelo Comitê de Ética em Pesquisa da Escola Superior de Educação Física da Universidade Federal de Pelotas-RS, sob o protocolo $n^{\circ}$ 073/2009. Todos os participantes concordaram em participar da pesquisa e assinaram o Termo de Consentimento Livre e Esclarecido.

\section{RESULTADOS}

Os dados sociodemográficos, de acordo com a tabela 1 , demonstram que a média de idade dos 263 participantes foi de 69,8 $\pm 6,44$ anos. Quando determinada por país, a média de idade

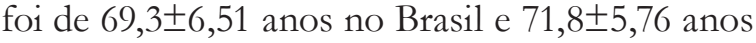
em Portugal. Isto demonstra que os idosos do PESEA eram um pouco mais velhos que os idosos do NATI $(p=0,01)$. Em relação à variável "gênero", foi constatado que havia mais homens praticando atividade física no PESEA $(22,0 \%)$ do que no NATI $(8,0 \%)$, diferença estatisticamente significativa $(\mathrm{p}=0,04)$.

Tabela 1. Descrição das variáveis sociodemográficas dos participantes nos projetos NATI (Brasil) e PESEA (Portugal). Pelotas-RS, 2010.

\begin{tabular}{lcccccc}
\hline \multicolumn{1}{c}{ Variável } & \multicolumn{2}{c}{ NATI } & \multicolumn{2}{c}{ PESEA } & \multicolumn{2}{c}{ Total } \\
& $\mathrm{n}$ & $\%$ & $\mathrm{n}$ & $\%$ & $\mathrm{n}$ & $\%$ \\
\hline Idade & & & & & & \\
60-69 anos & 119 & 55,9 & 20 & 40,0 & 139 & 52,9 \\
$70-79$ anos & 79 & 37,1 & 25 & 50,0 & 104 & 39,5 \\
$80-89$ anos & 15 & 7,0 & 05 & 10,0 & 20 & 7,6 \\
Gênero & & & & & & \\
Masculino & 17 & 8,0 & 11 & 22,0 & 28 & 10,6 \\
Feminino & 196 & 92,0 & 39 & 78,0 & 235 & 89,4 \\
\hline
\end{tabular}

Quanto aos motivos de adesão ao NATI e ao PESEA, conforme descrito na tabela 2 , verificam-se diferenças entre os idosos dos dois projetos. $\mathrm{Na}$ análise comparativa, houve diferença significativa para "indicação médica" entre o NATI (59,0\%) e o PESEA
$(26,0 \%),(p<0,001)$; "recuperação de lesões", NATI $(33,5 \%)$ e o PESEA $(12,0 \%),(\mathrm{p}=0,003)$; "ocupação do tempo livre", (28,8\% e 54,0\%), respectivamente, $\quad(\mathrm{p}<0,001) ;$ e "motivos estéticos" $(21,2 \%$ e 2,0\%), respectivamente $(\mathrm{p}=0,001)$. 
Tabela 2. Motivos de adesão ao projeto em relação a ganhos específicos em qualidade de vida. PelotasRS, 2010.

\begin{tabular}{lcc}
\hline \multicolumn{1}{l}{ Variável } & NATI & PESEA \\
& $\%$ & $\%$ \\
\hline Atividade no projeto & 64,0 & 4,0 \\
Ginástica & 40,1 & 74,0 \\
Hidroginástica & 7,5 & 42,0 \\
Musculação & & \\
Ano de ingresso & 56,9 & 54,0 \\
22005-2006 & 25,9 & 32,0 \\
2007-2008 & 17,6 & 14,0 \\
2009-2010 & & \\
Motivo de adesão ao projeto & 59,0 & $26,0 *$ \\
Indicação médica & 55,0 & 68,0 \\
Manutenção da flexibilidade & 33,5 & $12,0 \ddagger$ \\
Recuperação de lesões & 38,7 & 44,0 \\
Superação do estresse & 28,8 & $54,0 *$ \\
Ocupação do tempo livre & 21,2 & $2,0^{* *}$ \\
Motivos estéticos &
\end{tabular}

2005 = antes de 2006; 2010: sem novos ingressantes no NATI; ${ }^{*} \mathrm{p}<0,001 ;{ }^{*} \mathrm{p}=0,001 ; \mathrm{t}=0,003$.

A tabela 3 mostra os valores percentuais relativos à importância atribuída pelos participantes de ambos os projetos à prática de atividade física. Pode-se verificar que a grande maioria dos idosos apontou a prevenção de problemas de saúde como principal importância da prática de atividade física. Entre os idosos do NATI, o percentual foi de $76,6 \%$, e entre os portugueses do PESEA, esse número chegou a $82,0 \%$. Os motivos estéticos tiveram o menor percentual de respondentes, tanto para os idosos do PESEA como do NATI. Ao comparar os percentuais que tratam da importância atribuída à prática de atividade física, houve diferenças estatisticamente significativas entre os idosos do NATI e do PESEA, no que diz respeito à "recuperação de lesões", onde os participantes do projeto NATI tiveram percentual de $27,3 \% \mathrm{e}$ os idosos do PESEA, de 12,0\%, ( $p=0,002)$. Além disso, a diferença também ficou evidenciada na variável "motivos estéticos", na qual os idosos do NATI tiveram percentual de $19,6 \%$ e os portugueses do PESEA, $0,0 \%(p<0,001)$. 
Tabela 3. Importância atribuída à atividade física em relação a ganhos específicos em qualidade de vida. Pelotas-RS, 2010.

\begin{tabular}{lcc}
\hline \multicolumn{1}{c}{ Variável } & NATI & PESEA \\
& $\%$ & $\%$ \\
\hline Importância da atividade física & & \\
Prevenção de problemas de saúde & 76,6 & 82,0 \\
Manutenção da flexibilidade & 54,1 & 66,0 \\
Recuperação de lesões & 27,3 & $12,0^{* *}$ \\
Manutenção da saúde & 65,1 & 52,0 \\
Superação do estresse & 39,2 & 32,0 \\
Convívio social & 25,4 & 28,0 \\
Motivos estéticos & 19,6 & $0,0^{*}$ \\
\hline
\end{tabular}

${ }^{*} \mathrm{p}<0,001 ;{ }^{* *} \mathrm{p}=0,002$.

\section{DISCUSSÃO}

O presente estudo avaliou diferenças e semelhanças entre populações de idosos integrantes de programas de atividade física gratuitos de duas universidades conveniadas. Observou-se grande participação do gênero feminino (89,4\%), tanto no NATI $(92,0 \%)$ quanto no PESEA (78,0\%), o que também se evidencia em outros estudos. Dados semelhantes podem ser observados em um dos estudos de Mazo, ${ }^{13}$ no qual a amostra foi composta de $88,3 \%$ mulheres idosas e apenas $11,7 \%$ homens idosos; no estudo de Freitas et al., ${ }^{14}$ as mulheres totalizaram 69,2\% da amostra; e no estudo de Cardoso et al., ${ }^{15}$ as mulheres também eram maioria, com 84,6\% idosas e 15,4\% idosos. Um dos motivos para explicar essa diferença pode ser pelo diferencial de mortalidade, que determina uma vida média mais elevada para as mulheres. ${ }^{16}$ Além disso, deve-se considerar o tipo e horário das atividades oferecidas nos projetos e a possibilidade de tais fatores favorecerem a participação do público feminino. E ainda, os homens demonstram maior resistência aos cuidados de atenção primária em saúde, na sua conservação e medidas de prevenção. O Ministério da Saúde no Brasil alerta que estas barreiras podem ser socioculturais e institucionais, de acordo com a Política Nacional de Atenção Integral à Saúde do Homem lançada em 2008. ${ }^{17}$

Uma das questões também analisadas neste estudo foi o ano de ingresso dos participantes nos projetos (NATI/PESEA). A maioria dos idosos do NATI $(56,9 \%)$ e dos portugueses do PESEA (54,0\%) analisados ingressou em 2006, ou antes, deste período, o que significa um tempo de prática de atividade física de pelo menos quatro anos.

Em relação a esta questão, levantam-se algumas considerações. Os participantes permanecem nos projetos por estarem satisfeitos com os serviços prestados ou, ainda, por estarem conscientes dos benefícios da atividade física para a melhora da saúde e da qualidade de vida. Além disso, os grupos de convivência que oferecem uma diversidade de projetos (culturais, atividades físicas, atividades manuais, etc.) têm tido grande procura, tendo em vista o importante papel social que desempenham ao oferecer locais que possibilitam trocas de experiências, afetos e novas amizades. ${ }^{18}$ 
Salienta-se que as atividades de convivência realizadas, além da atividade física, acontecem somente no projeto PESEA, visto que o projeto NATI oferta somente atividades físicas aos idosos. No entanto, os resultados mostraram que o tempo de adesão aos projetos foi semelhante. Contudo, há outro ponto a considerar, que é a gratuidade desse tipo de serviço oferecido como projeto social por parte das universidades, uma vez que muitos participantes não possuíam condições financeiras para arcar com serviços privados, o pode estar relacionado à adesão aos projetos.

Alguns estudos têm procurado identificar os principais agentes que dificultam a adesão de indivíduos a programas de atividade física orientada. Falta de companhia, problemas de saúde, problemas financeiros, falta de tempo e falta de conhecimento sobre os benefícios da prática de atividade física são os mais citados, ${ }^{7,19-21}$ elucidando os resultados encontrados no presente estudo.

Analisando ainda os motivos de adesão aos projetos mais relatados pelos grupos, ficou demonstrado que o principal motivo para a prática de AF (atividade física) relacionado à saúde, relatado pelos idosos brasileiros do NATI, foi a "orientação médica" $(\mathrm{p}<0,001)$, motivo que encontra consistência na literatura, ${ }^{14}$ diferentemente dos portugueses do PESEA, que priorizaram a "manutenção da flexibilidade", diferença não significativa entre os grupos.

Loureiro $^{21}$ realizou estudo no qual 50 sujeitos voluntários (14 homens e 36 mulheres) que praticavam AF regular na cidade de Porto Alegre-RS, com idade superior a 60 anos, foram entrevistados com o objetivo de identificar os principais fatores que os levavam a praticar a $\mathrm{AF}$ regular. Os resultados obtidos mostraram que o principal motivo foi a busca pela qualidade de vida, seguido pela manutenção da saúde, semelhante ao apresentado no presente estudo.

Em estudo realizado por Rocha et al. ${ }^{22} \mathrm{com}$ idosos residentes em Itabuna-BA e vinculados a uma Unidade de Saúde da Família (USF), todos os participantes indicaram que a atividade física é extremamente importante para a saúde, e outros aspectos como controle do peso, aptidão física, humor, atratividade, divertimento ou harmonia receberam importância menor, corroborando o presente estudo.

Segundo Freitas et al., ${ }^{14}$ os principais motivos que levam os idosos a permanecer num programa de AF são: melhorar a postura (75\%); promover o bem-estar $(74,2 \%)$; manter-se em forma $(70,8 \%)$; sentir prazer $(66,7 \%)$; ficar mais forte e receber incentivos do professor (62,5\%); sentir bemestar provocado pelo ambiente (60\%); sentir-se realizado e receber atenção do professor (57,5\%). No estudo de Freitas et al., ${ }^{14}$ aparece uma variável um pouco diferente das apresentadas anteriormente: a relação com o professor.

Considera-se essa variável importante no processo de adesão ao projeto, e no presente estudo ela pode ter interferido nos resultados, uma vez que a troca de experiências entre gerações atua como agente motivador e pode influenciar positivamente na percepção que os idosos têm de sua vida. A grande maioria dos idosos não tem convivência no âmbito doméstico e consegue, por meio da participação no projeto, estabelecer relacionamentos e resgatar valores esquecidos. ${ }^{23}$

O processo motivacional que permeia a adesão dos idosos aos projetos é algo bastante complexo, que passa por inúmeras esferas social, cultural e biológica. Davidof ${ }^{24}$ acredita que a motivação se refere apenas a um estado interno e resultante de uma necessidade que ativa ou desperta comportamentos; ou seja, o indivíduo depende da própria vontade para realizar alguma tarefa ou atividade.

Já para Samulski, ${ }^{25}$ a motivação pode ser caracterizada como um processo ativo, internacional e dirigido a uma meta, o qual depende da interação de fatores pessoais (intrínsecos) e ambientais (extrínsecos). Desse modo, além da própria vontade, o indivíduo depende de inúmeros fatores relacionados ao contexto em que se insere. 
Traduzindo para a realidade de um programa de atividade física universitário, tais variáveis podem ser as relações sociais estabelecidas com os professores ministrantes das atividades e com os demais idosos participantes. Para o público da terceira idade, essa interação com o meio é muito importante, pois consegue melhorar seu autoconceito e percepção de bem-estar, na medida em que os idosos se sentem inseridos e participantes da sociedade. ${ }^{26}$

Apesar de algumas diferenças serem encontradas nas intenções dos idosos ao praticar AF orientada, uma constante pode ser observada - a forte relação entre AF e a busca por saúde, que demonstra a grande preocupação dos idosos em buscar formas de envelhecer com qualidade de vida. Por meio da participação em programas universitários de $\mathrm{AF}$, os idosos podem ter o conhecimento e a informação necessários para conseguir mudar seu estilo de vida e adotar hábitos mais saudáveis, por meio das instruções de profissionais qualificados.

Essa questão pode ser respaldada na medida em que políticas governamentais de saúde voltadas para a terceira idade, como a Active Ageing (Envelhecimento Ativo), desenvolvida pela Organização Mundial da Saúde, ${ }^{1}$ têm sido fomentadas, buscando propiciar um envelhecimento com mais saúde e qualidade de vida à população idosa.

Os resultados encontrados tornam-se fundamentais aos profissionais de Educação Física na tomada de decisões direcionadas a programas de intervenção em AF para a terceira idade. De acordo com Dishman ${ }^{27}$ (citado por Andreotti \& Okuma), faz sentido a promoção efetiva da AF pelo aconselhamento dos profissionais da saúde, visto que à medida que se envelhece, o contato com este tipo de profissionais aumenta. Segundo Falsarella \& Salve, ${ }^{28}$ a orientação dos professores de Educação Física durante as aulas é de extrema importância, pois a AF com o enfoque pedagógico, em que as informações são claras e objetivas, fundamentadas em princípios sociais, biológicos e psicológicos, proporciona aos idosos práticas corporais organizadas, planejadas e bem orientadas.

Segundo Cipriani et al., ${ }^{29}$ a prática de atividade física atua como importante fator para a manutenção da aptidão funcional. Além disso, a prática de atividade física regular parece reduzir os efeitos negativos acarretados pelo processo de envelhecimento, retardando inclusive algumas restrições quanto à realização das atividades de vida diária, prolongando o tempo de vida ativa dessa população. ${ }^{30}$

Nesse âmbito, as universidades, segundo Matsudo, ${ }^{31}$ estão entre os principais agentes sociais de programas direcionados à população idosa que oportunizam a participação em atividades físicas, intelectuais e sociais, o que garante um envelhecimento mais ativo, saudável e com melhor qualidade de vida para a população idosa.

O presente estudo apresentou algumas limitações, como o tamanho restrito da amostra, se considerar a realidade brasileira e portuguesa. Além disso, houve distribuição desigual, considerando os dois projetos. A amostra do projeto NATI (Brasil) é superior à do PESEA (Portugal), o que pode ter interferido em alguns resultados, como os relacionados à importância atribuída para a prática de atividade física ou os motivos de adesão ao projeto. $\mathrm{O}$ instrumento utilizado foi desenvolvido pelos pesquisadores, e não foi validado, representando outra limitação do estudo. Entretanto, os resultados encontrados exprimem uma importante realidade e possibilidade para o trabalho com a terceira idade.

Sugere-se a realização de mais estudos como este, visando compreender o perfil de usuários dos projetos de terceira idade, vinculados ou não a instituições de ensino superior, com a utilização de instrumentos validados, bem como considerando um tamanho de amostra representativo. Deste modo, é possível traçar um panorama mais fidedigno da realidade envolvendo a população idosa ativa. 


\section{CONCLUSÕES}

$\mathrm{O}$ estudo identificou diferenças significativas entre os grupos, relacionadas principalmente a variáveis sociodemográficas, nas quais os participantes portugueses (PESEA) apresentaram média de idade superior aos idosos brasileiros (NATI), bem como número superior de homens. Isso já era esperado, considerandose a diferença de desenvolvimento econômico e social dos países envolvidos. Entretanto, mesmo vivendo realidades culturais diferentes, os brasileiros do NATI e os portugueses do PESEA compartilham objetivos e motivação para a prática de atividade física.

Constatou-se, também, forte relação estabelecida entre atividade física e saúde, em ambos os projetos sociais estudados nos

\section{REFERÊNCIAS}

1. World Health Organization, Noncommunicable Disease Prevention and Health Promotion, Ageing and Life Course. Active ageing: a policy framework [Internet]. Geneva: WHO; c2002 [acesso 03 jul 2010]. 59 p. Disponível em http://www.who.int/ageing/ publications/active/en/index.html

2. Mazo GA, Lopes MA, Benedetti TB. Atividade física e o idoso: concepção gerontológica. 2. ed. Porto Alegre: Sulina; 2004. 236 p.

3. Gallahue DL, Ozmun JC. Compreendendo o desenvolvimento motor: bebês, crianças, adolescentes e adultos. 3. ed. São Paulo: Phorte; 2005.

4. Kohrt W, Bloomfield S, Little K, Nelson M, Yingling V. Physical activity and bone health". Med Sci Sports Exerc 2004;36(11):1985-96.

5. Bouchard C, Shephard RJ. Physical activity, fitness, and health: international proceedings and consensus statement. Champaign: Human Kinetics; 1994. 1055 p.

6. Warburton DE, Nicol CW, Bredin SSD. Health benefits of physical activity: the evidence. Can Med Assoc J 2006;174(6):801-9.

7. Dumith SC, Domingues MR, Gigante DP. Estágio de mudança de comportamento para a prática de atividade física: uma revisão literatura. Rev Bras Cineantropom Desempenho Hum 2008;10(3):301-7. dois países, visto que a principal importância atribuída à prática de atividade física regular foi a prevenção de doenças. Desse modo, os idosos têm mostrado que buscam, ao participar de programas de atividade física, alternativas para envelhecer com saúde e bem-estar.

Os governos devem procurar, por meio da elaboração de políticas públicas, fomentar a organização e criação de mais programas de atividades físicas voltados para a terceira idade, já que, atualmente, o elevado número de idosos é uma realidade e deve ter atenção especial. Além disso, sabendo dos benefícios que a atividade física proporciona à saúde, principalmente no que diz respeito à prevenção de doenças, os gastos com o sistema de saúde poderiam ser minimizados, proporcionando melhorias em outros setores da sociedade.

8. Reichert FF, Barros AJD, Domingues MR, Hallal PC. The role of perceived personal barriers to engagement in leisure-time physical activity. Am J Public Health 2007;97(3):515-9.

9. Hallal PC, Victora CG, Wells JCK, Lima RC. Physical inactivity: prevalence and associated variables in Brazilian adults. Med Sci Sports Exerc 2003;35(11):1894-900.

10. Siqueira FV, Facchini LA, Piccini RX, Tomasi E, Thumé E, Silveira DS, et al. Atividade física em adultos e idosos residentes em áreas de abrangência de unidades básicas de saúde de municípios das regiões Sul e Nordeste do Brasil. Cad Saúde Pública 2008;24(1):39-54.

11. Booth ML, Owen N, Bauman A, Clavisi O, Leslie E. Social-cognitive and perceived environment influences associated with physical activity in older Australians. Prev Med [Internet] 2000 [acesso 06 dez 2010];31(1):15-22. Disponível em: http://blast. activelivingbydesign.org/fileadmin/template/ documents/Booth_PreventiveMedicine_2000.pdf

12. Garcia T, Ramos MG, Afonso MR. "Vozes da Comunidade”. Mesa Redonda; 2009. Porto Alegre: UFRGS, Programa de Pós-Graduação em Educação; 2009. 
13. Mazo G, Cardoso F, Aguiar D. Programa de hidroginástica para idosos: motivação, auto-estima e auto-imagem. Rev Bras Cineantropom Desempenho Hum 2006;8(2):67-72.

14. Freitas CMSM, Santiago MS, Viana AT, Leão AC, Freyre C. Aspectos motivacionais que influenciam a adesão e manutenção de idosos a programas de exercícios físicos. Rev Bras Cineantropom Desempenho Hum 2007;9(1):92-100.

15. Cardoso AS, Borges LJ, Mazo GZ, Benedetti TB, Kuhnen AP. Fatores influentes na desistência de idosos em um programa de exercício físico. Rev Mov 2008;14(1):225-39.

16. Instituto Brasileiro de Geografia e Estatística. Perfil das mulheres responsáveis pelos domicílios no Brasil 2000 [Internet]. Rio de Janeiro: IBGE; 2002 [acesso 16 jul 2009]. (Estudos e Pesquisas, Informação Demográfica e Socioeconômica ;n.8). Disponível em: http://www.ibge.gov.br/home/estatistica/populacao/ perfildamulher/perfilmulheres.pdf

17. Ministério da Saúde (Brasil). Secretaria de Atenção à Saúde, Departamento de Ações Programáticas Estratégicas. Política nacional de atenção integral à saúde do homem: princípios e diretrizes [Internet]. Brasília (DF): Ministério da Saúde; 2008 [acesso 29 mar 2010]. Disponível em: http://www.saude.gov.br

18. Meirelles MEA. A atividade física na terceira idade. 3. ed. Rio de Janeiro: Sprint; 2000. 112 p.

19. Cassou ACN, Fermino RC, Santos MS, RodriguezAnêz CR, Reis RS. Barreiras para a atividade física em idosos: uma análise por grupos focais. Rev Educ Física 2008;19(3):353-60.

20. Bjornsdottir G, Arnadottir SA, Halldorsdottir S. Facilitators of and barriers to physical activity in retirement communities: experiences of older women in urban areas. Phys Ther 2012;92(4):551-62.

21. Loureiro LL. Motivação na prática de atividade física para pessoas na terceira idade. In: Anais do $10^{\circ}$ Seminário Intermunicipal de Pesquisa, $5^{\text {a }}$ Mostra de Atividades Extensionistas e Projetos Sociais, $8^{\circ}$ Salão de Iniciação Científica e Trabalhos Acadêmicos. Canoas: Editora da ULBRA, 2007. p. 1-22.

22. Rocha SV, Carneiro LRV, Costa-Júnior LOS. Motivos para a prática de atividade física entre idosos no município de Itabuna-BA. Rev APS 2011;14(3):276-82.

23. Bittar C, Lima LCV. O impacto das atividades em grupo como estratégia de promoção da saúde na senescência. Rev Kairós 2011;14(4):101-18.

24. Davidoff LL. Introdução à psicologia. 3 ed. São Paulo: Makron Books; 2001.

25. Samulski DM. M. Psicologia do esporte. Ed. Manole; 2002. 404 p.

26. Chodzko-zajko WJ, Proctor DN, Singh MA, Fiatarone M, Christopher T, Nigg CR, et al. Exercise and physical activity for older adults. Med Sci Sports Exerc 2009;41(7):1510-30.

27. Andreotti MC, Okuma SS. Perfil sócio-demográfico e de adesão inicial de idosos ingressantes em um programa de educação física. Rev Paul Educ Fís 2003;17(2):143-53.

28. Falsarella GR, Salve MGC. Envelhecimento e atividade física: Análise das relações pedagógicas professor/aluno. Integração 2006;6(47):345-50.

29. Cipriani NCS, Meurer ST, Benedetti TRB, Lopes MA. Aptidão funcional de idosas praticantes de atividades físicas. Rev Bras Cineantropom Desempenho Hum 2010;12(2):106-11.

30. Rosa MF, Mazo GZ, Silva AH, Brust C. Efeito do período de interrupção de atividades aquáticas na aptidão funcional de idosas. Rev Bras Cineantropom Desempenho Hum 2008;10(3):237-42.

31. Matsudo SM, Matsudo VKR, Barros Neto TL. Impacto do envelhecimento nas variáveis antropométricas, neuromotoras e metabólicas da aptidão física. Rev Bras Ciênc Mov 2000;8(4):21-32. 